\title{
Test difficulty and judgment bias
}

\author{
GREGORY SCHRAW and TERESA DEBACKER ROEDEL \\ University of Nebraska, Lincoln, Nebraska
}

\begin{abstract}
Two experiments tested the hypothesis that overconfidence in performance judgments is due to test- and person-driven errors. In Experiment 1, test difficulty accounted for the vast majority of variation in overconfidence when individuals judged items of varying difficulty within a homogeneous test. In Experiment 2, the severity of overconfidence did not differ between three unrelated tests once test difficulty was controlled. Both experiments supported the view that overconfidence is due largely to test difficulty. Some degree of overconfidence also occurred because individuals adopted a normatively high success criterion for judging their own test performance.
\end{abstract}

Previous research has suggested that people's ability to calibrate their performance may be related to the difficulty of a test (Pressley \& Ghatala, 1988) or the likelihood of a judged outcome (Lichtenstein \& Fischhoff, 1977). Typical calibration graphs of the relationship between judged and actual test performance like those presented in Baron (1988, pp. 199-200) and Fischhoff (1988, p. 173) reveal that judgment bias is more severe when subjects are judging the occurrence of frequent rather than infrequent events. In addition, overconfidence is more common than underconfidence and more likely to occur when a test is difficult (Cutler \& Wolfe, 1989; Newman, 1984). People also appear to be most overconfident when their confidence in a judgment is high to begin with (Vallone, Griffin, Lin, \& Ross, 1990). These patterns have been observed on a variety of tasks, including probability judgments (Fischhoff, 1988), reading comprehension (Glenberg, Sanocki, Epstein, \& Morris, 1987), recalling emotions (Thomas \& Diener, 1990), and social judgments (Dunning, Griffin, Milojkovic, \& Ross, 1990).

The fact that judgment bias may be related to performance difficulty has important implications for the interpretation of calibration research, since the overconfidence effect described above may be due in part to test difficulty (Lichtenstein \& Fischhoff, 1977). Our purpose in the present research was to investigate this relationship in further detail, focusing on differences between testdriven and person-driven sources of error. We argue that calibrating one's performance may be construed as a probabilistic event in which systematic bias is introduced as a function of test difficulty (see Baron, 1988, pp. 198-204 for a further explanation). For example, when $80 \%$ of the items on a test are answered correctly, the probability of underestimating one's performance should be greater than that of overestimating it, since there is a

Correspondence regarding this manuscript should be sent to G. Schraw, Department of Educational Psychology, 1313 Seaton Hall, University of Nebraska, Lincoln, NE, 68588-0641.

-Accepted by previous editor, Margaret Jean Intons-Peterson greater chance of erring in one direction (i.e., underconfidence). When an item is answered correctly half the time, judgment errors should be less severe, since overand underconfidence are equally likely to occur.

One advantage to thinking about calibration probabilistically is that it provides a method for distinguishing between different kinds of judgment errors. Bias that is due to a test's difficulty reflects a form of test-driven error, since judgments will be biased in different directions, depending upon the test's difficulty. In contrast, bias due to an inability to assess accurately one's performance reflects person-driven error. We argue that both kinds of error can be distinguished by comparing test-takers' calibration across tests that range in difficulty. If poor calibration is due to test-driven rather than person-driven factors, one would expect easy tests to lead to underconfidence, difficult tests to lead to overconfidence, and moderately difficult tests to lead to very little random error. Calibration errors that diverge from this pattern should reveal the degree to which poor calibration can be attributed to person-driven error components such as persistent overconfidence.

Two experiments were conducted to investigate the conditions under which systematic bias occurs. Bias was measured by taking the difference between average performance and average estimated performance for a set of questions and was used as an index of the direction of judgment error (Yates, 1990, pp. 61-63). Bias ranged in the present studies from +100 to -100 , with a score of zero indicating no bias, a score greater than zero indicating overconfidence, and a score less than zero indicating underconfidence. Bias was not measured using correlational measures such as Pearson's $r$ or gamma (Nelson, 1984), for several reasons. First, measures of association are insensitive to the magnitude of the difference between two sets of scores (Aldenderfer \& Blashfield, 1984) and therefore do not provide a reliable measure of over- and underconfidence. For example, imagine four people who weigh $120,130,140$, and 150 pounds, respectively, and that Judge A estimates their weights at $130,140,150$, and 160 pounds, while Judge B estimates their weights at 123 , 126,135 , and 162 pounds. In this case, the correlation 
between true and judged weight is higher for Judge $A$ than for Judge B, even though the former's estimates are more biased. A second difficulty with correlations is that a negative correlation need not correspond to underconfidence. For example, Judge C may predict weights of 220,200 , 180 , and 160 pounds, respectively, which yield a negative correlation despite consistent overconfidence.

The probabilistic model described above predicts that overconfidence increases as test difficulty increases. Thus, the critical examination of test- versus person-driven bias can be decided in a straightforward way by comparing whether the same judges are consistently overconfident or whether the direction of their judgment bias changes as a function of test difficulty. The degree to which judges are overconfident on all tests provides a measure of person-driven bias (i.e., the overconfidence effect); the degree to which overconfidence occurs only on difficult tests provides a measure of test-driven bias.

We also examined whether judgment errors follow a linear or nonlinear trend across different levels of test difficulty. A strong linear trend suggests that errors are directly proportional to test difficulty; a nonlinear trend suggests that errors increase exponentially as a test becomes increasingly easy or difficult; that is, judgment bias is compounded when a test becomes extremely easy or difficult. In the present research, we made no specific assumptions about the underlying distribution of judgment errors-for example, about whether errors were modeled best by a uniform or a normal distribution. However, the trend between judgment errors and test difficulty was expected to be linear. Evidence of a strong linear trend is most consistent with the hypothesis that judgment error is due primarily to test difficulty. Curvilinear trends are consistent with the hypothesis that person-driven error contributes to judgment errors over and above the effect of test difficulty.

\section{EXPERIMENT 1}

Our purpose in Experiment 1 was to investigate whether judgment bias changes as a function of test difficulty. To do so, we examined judgment errors across varying levels of item difficulty within the same reading comprehension test. Test items were divided into three levels of difficulty; easy, moderately difficult, and difficult. We predicted that judges would be underconfident on easy items, overconfident on difficult items, and unbiased on moderately difficult items. These predictions reflect the assumption that judgment error is due principally to the difficulty of a test rather than to person-driven error. Finding that overconfidence decreases in magnitude as test items become easier would support the test-driven error hypothesis. Finding that overconfidence increases as items become easier or remains constant across items of different levels of difficulty would support the person-driven error hypothesis.

\section{Method}

Participants. Forty-eight undergraduates enrolled in an introductory educational psychology class participated as part of their normal course assignment.
Materials. The materials consisted of the Nelson-Denny Reading Comprehension Test (Form E) (Brown, Bennett, \& Hanna, 1981). The first of the eight passages included in this test was used as a warm-up; the remaining seven were used as experimental materials. The seven passages included 28 five-option multiple-choice test questions that were divided into three levels of difficulty based on data gathered by Schraw and Roedel (1992); those falling in the $30 \%-50 \%$ difficulty range, those in the $50 \%-70 \%$ range, and those in the 70\%-90\% range. The three difficulty levels included 5,12 , and 10 items, respectively; 1 item was excluded that was answered with greater than $90 \%$ accuracy.

Procedure. Subjects participated in two groups of 23 and 25 individuals. The instructions were identical for both groups. Individuals first read experimental instructions from the cover of their test booklets and then received a verbal reiteration of these instructions. The instructions stated that the purpose of the study was to examine how well individuals could assess their test performance. The subjects were allowed to proceed through the tests at their own pace, but they were not allowed to leave the testing room until everyone had completed the test and was debriefed. The instructions specified that eight different tests were included in the test booklets and that the first was a practice passage. The subjects were told to study each passage as long as they wanted, complete the corresponding test items, rate how much confidence they had in each response, and then proceed to the next passage. Test questions and rating scales for each of the eight passages appeared on a separate sheet. The subjects were not allowed to look back at the target passage once they began the test.

Confidence ratings were made on a $100-\mathrm{mm}$ rating scale adapted from Schiffman, Reynolds, and Young (1981). The left end of this scale was labeled $0 \%$ confidence, while the right end was labeled $100 \%$ confidence. The participants made their responses by drawing a slash on the part of the scale that best corresponded to their perceived confidence. Bias was computed by taking the difference between the average performance score and average estimated performance score for all items on each of the easy, moderately difficult, and difficult question sets. This led to three mean bias scores that reflected the average degree of bias at each level of difficulty (Rushton, Brainerd, \& Pressley, 1983). ${ }^{1}$ Mean scores were used as the unit of analysis to minimize the effect of low reliability when items are analyzed separately (Weaver, 1990).

\section{Results}

Performance (i.e., actual item-set score), confidence (i.e., estimated item-set score), and bias (i.e., the difference between these scores) were analyzed separately in oneway repeated measures analyses of variance (ANOVAs). The means and standard deviations for these scores across the three item-sets appear in Table 1. All tests for this experiment and for Experiment 2 were significant at the $p<.05$ level unless otherwise noted.

Individual ANOVAs revealed a significant main effect of test difficulty, using performance $[F(2,94)=53.13$, $\left.M S_{\mathrm{e}}=1.92\right]$ and confidence $\left[F(2,94)=17.10, M S_{\mathrm{e}}=\right.$ $.212]$ as dependent variables. A post hoc analysis of these means using Dunn's multiple comparison procedure based on three comparisons (Kirk, 1982, pp. 106-111) showed that difficult items $(M=36.41, S D=24.87)$ were answered less accurately than moderately difficult $(M=$ $59.44, S D=22.43)$ or easy $(M=76.35, S D=18.85)$ items $[t(141)=81.37$ and 141.13 , respectively $]$. In addition, the moderately easy items were recalled less accurately than the easy items $[t(141)=57.75]$. Confidence ratings on difficult items $(M=63.63, S D=24.35)$ were lower than on moderately difficult $(M=75.41, S D=$ 
Table 1

Means and Standard Deviations for Performance, Confidence, and Bias Scores in Experiment 1

\begin{tabular}{|c|c|c|c|c|c|c|}
\hline \multirow[b]{3}{*}{ Score } & \multicolumn{5}{|c|}{ Items } & \\
\hline & \multicolumn{2}{|c|}{ Easy } & \multicolumn{2}{|c|}{$\begin{array}{l}\text { Moderately } \\
\text { Difficult }\end{array}$} & \multicolumn{2}{|c|}{ Difficult } \\
\hline & $M$ & $S D$ & $M$ & $S D$ & $M$ & $S D$ \\
\hline Performance & 76.35 & 18.85 & 59.44 & 22.43 & 36.41 & 24.87 \\
\hline Confidence & 74.80 & 27.31 & 75.41 & 22.69 & 63.63 & 24.35 \\
\hline Bias & -1.55 & 15.65 & 15.97 & 21.98 & 27.22 & 29.70 \\
\hline
\end{tabular}

Note-Performance and confidence scores are expressed as proportions. Bias scores range from -100 to +100 .

22.69) or easy items $(M=74.80, S D=27.31)[t(141)=$ 126.66 and 120.10 , respectively].

The main effect of item difficulty was significant as well in a comparison of bias scores $\left[F(2,94)=24.17, M S_{\mathrm{e}}=\right.$ 1.00]. Bias was greater on difficult items $(M=27.22$, $S D=29.70)$ than on moderately difficult $(M=15.97$, $S D=21.97)$ or easy $(M=-1.55, S D=15.65)$ items $[t(141)=56.26$ and 143.85 , respectively $]$. Moderately difficult items were more biased than easy items as well $[t(141)=87.60]$. A test of this trend using procedures described by Winer (1971, pp. 296-301) revealed it to be due almost entirely to the linear trend component, which accounted for $95 \%$ of the total sample variance $[F(1,47)=$ $42.65, p<.001]$.

\section{Discussion}

The results of Experiment 1 supported our main prediction that bias was related to test item difficulty. Changes in the degree of overconfidence across different levels of item difficulty indicated that overconfidence was a byproduct of test difficulty; the easier an item, the less overconfident subjects were when judging their performance on these items. A strong linear trend in bias scores further supported this conclusion; as difficulty increased, bias increased in proportion to it. These findings are at odds with the view that human judgment is predisposed to gross overconfidence (cf. Vallone et al., 1990).

However, our findings also were at odds with our predictions, in that easier items led to no discernible bias even though a significant degree of underconfidence was expected to occur by chance. Moderately difficult items also led to a significant degree of overconfidence, even though these items were expected to minimize bias. Errors of this kind suggest some degree of person-driven bias that cannot be attributed to test difficulty alone. An inspection of the mean bias scores in Table 1 suggests that test-takers adjusted their confidence ratings on the basis of perceived normative difficulty of the test; that is, they relied on implicit base rates and expectations about performance to weight their judgments. For example, Table 1 indicates that easy and moderately difficult items led to confidence ratings that were approximately equal to the normative difficulty of the entire test. On a single, homogeneous test like the one used here, test-takers may base their confidence judgments to a large degree on such an estimate even though it may adversely affect their ability to calibrate their performance.

Overall, Experiment 1 reported that overconfidence in a typical calibration-of-performance task is attributable largely to the difficulty of a test item, with the proviso that error may be introduced as well whenever judges use a success base rate that deviates from $50 \%$ difficulty. In the present case, participants apparently expected to do rather well on the test, which resulted in the point at which zero bias occurred being $75 \%$ correct rather than $50 \%$ correct. However, we found no evidence of persistent overconfidence across different levels of difficulty even though individuals tended to be somewhat overconfident on moderately difficult items and highly overconfident on difficult items. Thus, there is little reason to believe that test-takers are predisposed to universally poor judgment. Rather, poor judgments are likely to occur under certain testing conditions.

\section{EXPERIMENT 2}

In Experiment 1, judgment error varied systematically as a function of test item difficulty within a homogenous test. Another potential source of judgment error is domain familiarity. In Experiment 2, we investigated this possibility by comparing performance judgments across three different domains, including reading comprehension, calculating simple probabilities, and estimating the length of a line given a scale model. Test difficulty was held constant at $50 \%$ difficulty, to control for differences in performance within these domains. Of primary interest was whether judgment errors differed across domains once test difficulty was eliminated as a confounding variable.

One hypothesis is that judgment errors are affected by one's ability to perform competently in a particular domain (see Lichtenstein, Fischhoff, \& Phillips, 1982, for a review) or by specific task constraints in that domain (Pressley \& Ghatala, 1990). For example, Pressley and Ghatala (1988) found calibration differences in tests of opposites and analogies in comparison with reading comprehension, even though these tests did not differ in difficulty. This finding implies that some tasks require a greater degree of calibration skill than others do, or that they are more prone to person-driven error than others are. An alternative explanation is that domain expertise 
or task differences are independent of judgment errors. Morris (1990), for example, reported that domain familiarity is independent of calibration even though it is not independent of test performance or the availability of testrelevant information in memory. Given this view, judgment bias should not differ across different domains once test difficulty is controlled.

We predicted that judgment errors are not significantly related to the type of test one takes, provided that those tests are of comparable difficulty; thus, we did not expect statistical differences to occur across the three domains regarding the magnitude of bias. This prediction is consistent with the assumption that judgment errors are attributable to test-driven rather than person- or taskdriven error. It also is consistent with Morris's (1990) findings that domain expertise does not constrain judgment accuracy to any significant extent.

\section{Method}

Subjects. Thirty-five undergraduates enrolled in an introductory educational psychology class participated in the study for extra credit.

Materials. Test booklets were arranged that included tests of reading comprehension, spatial judgments, and probability questions. These three tests were selected from a pool of nine tests used previously by Schraw and Roedel (1992). The three tests used in Experiment 2 were selected so that (1) overall test difficulty would be held constant at roughly $50 \%$ and (b) the three tests would measure performance in three conceptually different domains.

The reading comprehension portion included Passages 5 (i.e., Fungi) and 7 (i.e., Painters) from the Nelson-Denny reading comprehension test (Form E) (Brown et al., 1981). After each passage, the participants answered four five-option multiple-choice questions for a total of eight questions. The remaining tests were prepared by the authors. Sample items from the probability and spatial judgment tests are included in the Appendix. Each of these tests included eight four-option multiple-choice test questions. The test booklets were constructed in such a way that each story in the reading comprehension phase was followed by a performance test and a single posttest confidence scale, each on a separate page. The remaining tests were presented, four questions per page, with a single confidence scale appearing at the end of the test.

Unlike in Experiment 1, estimated performance accuracy was measured on a single five or nine-point scale for each set of test items. For example, the eight-question probability test was followed by a 9-point scale labeled 0 correct, 1 correct, 2 correct, etc. Zero correct corresponded to $0 \%$ confidence, 4 correct corresponded to $50 \%$ confidence, etc. The participants responded by circling the number correct that best corresponded to their perceived confidence. A single measure of confidence was used in this experiment, since it provided a more direct estimate of average performance over the entire set of items in comparison with the individual scales used in Experiment 1. ${ }^{2}$

Procedure. The subjects participated in two groups of 20 and 15 people. The instructions were identical for both groups. The participants first read the experimental instructions from the cover of their test booklets and then received a verbal reiteration of these instructions. The instructions stated that the purpose of the study was to examine how well individuals could assess their test performance in unrelated domains. The subjects were allowed to proceed through the tests at their own paces, but they were not allowed to leave the testing room until everyone had completed the test and was debriefed. The instructions specified that three different tests were included in the test booklets. The participants were told to study the stimulus materials for as long as they wanted, complete the corresponding test items, and then rate how many test items they had answered correctly. As in Experiment 1, bias was computed by taking the difference between average performance and estimated performance on each test.

\section{Results and Discussion}

Performance, confidence, and bias scores were analyzed separately in one-way repeated measures ANOVAs. All means and standard deviations appear in Table 2.

Three findings warrant discussion. First, performance accuracy was approximately $50 \%$ on each of the three tests and did not differ significantly, as predicted. Second, a significant difference was found for confidence ratings $\left[F(2,68)=8.07, M S_{\mathrm{e}}=.017\right]$. A post hoc analysis, using Dunn's multiple comparison procedure with three comparisons, showed that confidence judgments for the probability test $(M=60.01, S D=19.61)$ were significantly lower than the corresponding judgments for reading comprehension $(M=72.01, S D=14.55)$ and spatial judgments $(M=70.63, S D=18.69)[t(102)=3.87$ and 3.22, respectively]. Third, no significant difference was found across the three tests when bias was used as the criterion variable. Overconfidence on each test corresponded to the same degree of overconfidence for moderately difficult items in Experiment 1.

The last of these findings reveals that judgment bias is relatively stable across widely different types of tests, provided that the difficulty of these tests is held constant. This pattern is consistent with our main prediction that bias is due in large part to test-driven rather than person- or task-driven judgment error and is compatible as well with Morris's (1990) finding that domain familiarity is independent of calibration ability. These findings also suggest that individuals calibrate in different domains with a high degree of consistency; that is, the degree to which an in-

Table 2

Means and Standard Deviations for Performance, Confidence, and Bias Scores in Experiment 2

\begin{tabular}{|c|c|c|c|c|c|c|}
\hline \multirow[b]{2}{*}{ Score } & \multicolumn{2}{|c|}{$\begin{array}{c}\text { Reading } \\
\text { Comprehension }\end{array}$} & \multicolumn{2}{|c|}{ Probability } & \multicolumn{2}{|c|}{$\begin{array}{c}\text { Spatial } \\
\text { Judgments }\end{array}$} \\
\hline & $M$ & $S D$ & $M$ & $S D$ & $M$ & $S D$ \\
\hline Performance & 50.03 & 23.96 & 47.08 & 25.52 & 52.52 & 17.54 \\
\hline Confidence & 72.01 & 14.55 & 60.01 & 19.61 & 70.63 & 18.69 \\
\hline Bias & 21.98 & 24.00 & 12.93 & 30.49 & 18.11 & 24.51 \\
\hline
\end{tabular}

Note-Performance and confidence scores are expressed as proportions. Bias scores range from -100 to +100 . 
dividual calibrates without bias may be a general rather than domain-specific skill.

A second finding was that individuals differed with respect to the degree of confidence that they had in their performance. Individuals expressed significantly less confidence when judging their performance on the probability test as opposed to the reading comprehension or spatial comparison tests. This suggests that individuals may be somewhat more cautious when judging performance in less familiar domains regardless of test difficulty, a difference that we attribute to test-takers' awareness of limited knowledge in that domain. Less confidence did not translate into significantly less bias, however, in part due to lower performance scores for probability questions, and in part due to the amount of variation in bias scores.

Our findings also suggested that the magnitude of bias did not differ between Experiments 1 and 2, despite the use of different measurement scales. An inspection of Table 1 reveals that bias for items with a mean difficulty level of $59 \%$ was 15.97 . The average difficulty level of all tests in Experiment 2 was $49 \%$, with a mean bias score of 18.06. In comparison, solving for a difficulty level of $50 \%$ in Experiment 1 with the use of multiple regression techniques produced a mean bias estimate of 19.21 $(S D=22.37)$. Thus, both measurement strategies led to virtually identical estimates of bias, given a comparable level of difficulty.

Last, our findings were at odds with those of Pressley and Ghatala (1988), who reported a large difference in the correlation between test performance and estimates of performance on tests of roughly equal difficulty. Specifically, calibration performance was lower on a test of reading comprehension than on a test of opposites and analogies. We attribute this difference to the way in which calibration was measured in these studies. In the present investigation, we examined the magnitude of judgment error (i.e., the difference between true and estimated performance), whereas Pressley and Ghatala examined the linear association between these scores. The disparity between difference scores and correlations may occur because the correlation between two variables need not correspond to the degree of bias between performance and estimated performance judgments. In our opinion, the findings presented by Pressley and Ghatala measure a separate, though equally important, component of calibration in comparison with our own. Their study indicates that test performance is a poor predictor of estimated performance on the reading comprehension test as opposed to the opposites and analogy tests. Our findings suggest that individuals were biased to an equal degree across tests, regardless of the correlation between test performance and estimated performance.

Overall, Experiment 2 showed that calibration ability across unrelated domains is stable, provided that one controls for the difficulty of test items within those domains. This suggests that the ability to calibrate one's performance is relatively stable. Experiment 2 also replicated the finding reported in Experiment 1 that test items of
$50 \%$ difficulty lead to overconfidence. Judges appeared to adopt a subjective criterion in which a $75 \%$ success rate on test questions was judged to be the norm. Items that were more difficult than this standard led to increasing degrees of overconfidence.

\section{GENERAL DISCUSSION}

The present research suggested three main conclusions that warrant further discussion: (1) the severity of overconfidence in calibration judgments can be accounted for in large part by test difficulty; (2) the severity of overconfidence is independent of content domain, and (3) individuals are overconfident in part because they adopt a higher than chance (i.e., $50 \%$ difficulty) success criterion when judging their test performance. These findings support and extend the previous work of Lichtenstein and Fischhoff (1977).

Consistent with our predictions, the magnitude of judgment error was a near perfect linear function of test item difficulty. When test item difficulty increased, overconfidence increased as well. When test items were easy, judgment errors were minimized. We found no evidence of persistent overconfidence, nor were individuals consistent in the degree of overconfidence across different levels of test difficulty. These patterns supported our main hypothesis that overconfidence is a function of test item difficulty rather than inherently poor human judgment, as suggested by a number of previous studies (Dunning et al., 1990; Vallone et al., 1990). Moreover, since Experiment 1 compared estimates of performance within subjects on a homogeneous task, it is unlikely that gross overconfidence on difficult items can be attributed to the nature of the task or to poor judgment on the part of judges.

We also found that the severity of overconfidence does not change as a function of the type of test one receives, provided those tests are of equal difficulty. This finding is consistent with previous work by Morris (1990), who reported that domain knowledge plays little role in calibration even though it is related strongly to access to testrelevant information in memory. It also suggests that monitoring one's performance may be a stable cognitive ability once difficulty is controlled. This conclusion is consistent with the work of Schraw and Roedel (1992), who reported that a significant percentage of college students calibrated with a high degree of accuracy (i.e., showed little bias) across eight unrelated domains. Combined, these studies suggest the presence of a general monitoring skill that varies between individuals, but varies little within individuals.

A third finding was that some degree of overconfidence in one's performance may be tied to expectations about performance. In both Experiments 1 and 2, overconfidence increased because performance judgments were made in relation to a $75 \%$ accuracy criterion rather than the $50 \%$ difficulty standard assumed by the probabilistic model described earlier. These findings indicate that test- 
takers use prior knowledge about tests to predict their overall performance and confidence. The degree to which they do so may introduce bias. When test difficulty matches this criterion, little bias should occur. When a test is considerably more difficult than this criterion (as in Experiment 2), a significant degree of bias may occur. Future studies are needed, to investigate the degree to which students are predisposed to adopt a particular success criterion, and in turn, how that criterion affects bias.

The present research suggests a number of guidelines for calibration researchers. First, calibration studies should take into account the difficulty of a task, since the more difficult the task, the more likely it is that test-takers will be overconfident. Second, researchers must bear in mind that the situational constraints of a calibration study affect both test- and person-driven sources of error and their interpretation. Manipulating the difficulty of the text affects the former; manipulating expectations of test difficulty affects the latter. Affecting perceptions of base rate may have an especially profound effect on one's results. Test-takers led to believe a test is easy, or those who assume a test will be easy without being provided any information, are more apt to be overconfident when a test proves to be difficult. Informing participants about the relative difficulty of a test may improve their calibration performance in several ways. One is that judgments of performance should be less biased. A second is that performance and judged performance scores should be more consistent, both within and between two sets of judgments, leading to a larger value of $r$ and gamma (Nelson, 1984).

In addition, researchers should exercise caution when interpreting results that appear to be similar at first glance. For example, a moderate degree of overconfidence on an easy test suggests poor calibration, since overconfidence should not occur by chance. A moderate degree of overconfidence on a difficult test, however, would be expected to occur by chance, and therefore should not be taken as a sign of poor calibration. A similar argument applies to underconfidence on easy and difficult tests.

Last, further research is needed to compare correlational and "difference" measures of calibration. Earlier, we suggested that the two provide somewhat different measures of the calibration process. One example of this dissimilarity is that calibration judgments may be highly accurate even though performance and estimated performance scores are uncorrelated. ${ }^{3}$ Presently it is unclear whether each measure is affected by test difficulty to the same degree, or whether one index is more susceptible to perturbation than another. Future studies should examine the performance of each measure under different testing conditions to clarify these uncertainties.

\section{REFERENCES}

Aldenderfer, M. S., \& Blashfield, R. K. (1984). Cluster analysis. Beverly Hills: Sage.

BARON, J. (1988). Thinking and deciding. Cambridge: Cambridge University Press.

Brown, J. E., Bennett, J. M., \& Hanna, G. (1981). The NelsonDenny Reading Test: Form E. Chicago: Riverside Publishing.
CUTLER, B. L., \& WOLFE, R. N. (1989). Self-monitoring and the association between confidence and accuracy. Journal of Research in Personality, 23, 410-420.

Dunning, D., Griffin, D. W., Milojkovic, J. D., \& Ross, L. (1990). The overconfidence effect in social prediction. Journal of Personality \& Social Psychology, 58, 568-581.

FisCHHOFF, B. (1988). Judgment and decision making. In R. Sternberg \& E. Smith (Eds.), The psychology of human thought (pp. 153-187). Cambridge: Cambridge University Press.

Glenberg, A. M., SanockI, T., Epstein, W., \& Morris, C. (1987). Enhancing calibration of comprehension. Journal of Experimental Psychology: General, 116, 119-136.

KIRK, R. E. (1982). Experimental design (2nd ed.). Belmont, CA: Brooks/Cole.

Lichtenstein, S., \& FischHoff, B. (1977). Do those who know more also know more about how much they know? Organizational Behavior \& Human Performance, 20, 159-183.

Lichtenstein, S., Fischroff, B., \& Phillips, B. (1982). Calibration of probabilities: The state of the art to 1980 . In D. Kahneman, P. Slovic, \& A. Tversky (Eds.), Judgment under uncertainty: Heuristics and biases (pp. 306-334). Cambridge: Cambridge University Press.

MORRIs, C. C. (1990). Retrieval processes underlying confidence in comprehension judgments. Journal of Experimental Psychology: Learning, Memory, \& Cognition, 16, 223-232.

Nelson, T. O. (1984). A comparison of current measures of the accuracy of feeling-of-knowing predictions. Psychological Bulletin, 95, 109-133.

Newman, R. S. (1984). Children's numerical skill and judgments of confidence in estimation. Journal of Experimental Child Psychology, 37, 107-123.

Pressley, M., \& Ghatala, E. S. (1988). Delusions about performance on multiple-choice comprehension tests. Reading Research Quarterly, 23, 454-464.

Pressley, M., \& Ghatala, E. S. (1990). Self-regulated learning: Monitoring learning from text. Educational Psychologist, 25, 19-33.

Rushton, J. P., Brainerd, C. J., \& Pressley, M. (1983). Behavioral development and construct validity: The principle of aggregation. Psychological Bulletin, 94, 18-38.

SchiffMan, S. S., ReYnolds, M. L., \& Young, F. W. (1981). Introduction to multidimensional scaling. New York: Academic Press.

SCHRAW, G., \& ROEDEL, T. D. (1992). An analysis of general metacognitive monitoring skills. Paper presented at the annual meeting of the American Educational Research Association, San Francisco.

Thomas, D. L., \& Diener, E. (1990). Journal of Personality \& Social Psychology, 59, 291-297.

VAllone, R. P., GRIFFin, D. W., LiN, S., \& Ross, L. (1990). Overconfident prediction of future actions and outcomes by self and others. Journal of Personality \& Social Psychology, 58, 582-592.

WEAVER, C. A. (1990). Constraining factors in calibration of comprehension. Journal of Experimental Psychology: Learning, Memory, \& Cognition, 16, 214-222.

WINER, B. J. (1971). Statistical principles in experimental design (2nd ed.). New York: McGraw-Hill.

YATES, F. (1990). Judgment and decision making. Englewood Cliffs, NJ: Prentice-Hall.

\section{NOTES}

1. It should be noted that each mean bias score consisted of the difference between two interval scale scores, even though test performance for each item was scored ordinally.

2. The authors wish to thank Charles A. Weaver III for suggesting this scoring change.

3. Consider the case in which eight objects weigh $100,110,120$, $130,140,150,160$, and 170 pounds each, and Judge A predicts weights of $130,140,120,100,170,120,130$, and 140 , while Judge $B$ predicts weights of $140,150,160,170,180,190,200$, and 210 . In this case, Judge $A$ is more accurate (i.e., average bias $=-4$ pounds) even though true and predicted weights are correlated at $r=.06$. In contrast, Judge B is less accurate (average bias $=+40$ pounds), despite a perfect correlation of $r=1.0$ between true and predicted weights. 


\section{APPENDIX \\ Sample Test Items Used in Experiment 2}

Mathematical Word Problems

1. The odds of a baby being a girl is $50 \%$. What is the probability of a couple having four consecutive girls out of four children?

$$
\text { A }(6 \%) \quad \text { B (12\%) } \quad \text { C (24\%) } \quad \text { D (50\%) }
$$

\section{Spatial Judgments}

1. How long is Line B when measured in feet?

A: (320 feet)
A (96)
B (128)
C (158)
D (186)

(Manuscript received October 1, 1992;

revision accepted for publication June 22, 1993.) 